\title{
Role of elastography strain ratio and TIRADS score in predicting malignant thyroid nodule
}

\author{
Hussein Hassan Okasha' \\ https://orcid.org/0000-0002-0815-1394 \\ Mona Mansor ${ }^{1}$ \\ https://orcid. org/0000-0002-7274-5429 \\ Nermine Sheriba ${ }^{2}$ \\ https://orcid. org/0000-0002-1222-8948 \\ Maha Assem ${ }^{1}$ \\ https://orcid. org/0000-0003-3295-2586 \\ yasmine abd elfatah ${ }^{1}$ \\ https://orcid.org/0000-0002-5302-4087 \\ Omar A. Ashoush' \\ https://orcid.org/0000-0003-3992-9856 \\ Maha Rakha' \\ https://orcid.org/0000-0002-8287-7599 \\ Dalia Abdelfatah ${ }^{3}$ \\ https://orcid.org/0000-0002-8598-7128
}

Shereen Sadik El-Sawy

https://orcid org/0000-0001-9642-4657

Mai Elshenoufy'

https://orcid. org/0000-0002-9781-9463

Ahmed Amr Mohsen ${ }^{4}$

https://orcid.org/0000-0003-3893-1524

Heba Kamal Sedrak'

https://orcid.org/0000-0001-9143-3247

Abeer Awad Abdellatif ${ }^{1}$

https://orcid.org/0000-0001-9945-9767

\begin{abstract}
Objective: Ultrasonography (US) is the most accurate and cost-effective imaging method in diagnosis of thyroid nodules. A practical thyroid imaging reporting and data system (TIRADS) for thyroid nodules has been proposed to classify nodules of the thyroid gland to solve the problem of nodule selection for fine needle aspiration cytology (FNAC). Real-time elastography and strain ratio (SR) is a method used to assess the stiffness and predict the malignancy of thyroid nodules. The objective of this study was to assess the role of elastography and SR and the TIRADS scoring system in discriminating malignant from benign thyroid nodules. Materials and methods: From 2015 to 2018 at Cairo University Hospital, a series of 409 patients with thyroid nodules was referred to undergo thyroid ultrasound. Categorization of each nodule according to the TIRADS ranged from 1 to 5 . The qualitative elastography score and semiquantitative SR of the nodules were evaluated. Final diagnosis was done by either postthyroidectomy histopathological examination or US-guided FNAC. Results: Our study included 409 patients with thyroid nodules. Their mean age was $39 \pm 10$ SD; 36 were males and 373 were females. There were 22 malignant nodules and 387 benign nodules. There were statistical differences between benign and malignant nodules regarding TIRADS classification, SR, anteroposterior/transverse ratio, degree of echogenicity, border, presence of calcification, and absence of halo sign $(P<0.001)$. The elastic properties of thyroid nodules proved to be a good discriminator between malignant and benign nodules $(P-<0.001)$ at a cut off value of $>2.32$ with $95.2 \%$ sensitivity and $86.5 \%$ specificity. For every unit increase in SR, the risk of malignancy increased by nearly 2 times. Patients with irregular borders had nearly 17 times increased risk of malignancy than those with regular borders. Conclusion: Elastography and SR proved to be of high significant value in discriminating benign from malignant nodules, so we recommend adding it to the TIRADS classification. Arch Endocrinol Metab. 2020;64(6):735-42
\end{abstract}

Keywords

Thyroid nodules; Elastography; TIRADS
${ }^{1}$ Kasr Al-Aini Hospitals, Cairo University, Cairo, Egypt

${ }^{2}$ Ain Shams University, Cairo, Egypt

${ }^{3}$ Biostatistic and Cancer

Epidemiology Department, National Cancer Institute, Cairo

University, Cairo, Egypt

${ }^{4}$ National Research

Centre, Cairo, Egypt
Correspondence to: Abeer Awad Abdellatif Internal Medicine Department, Kasr Al-Aini Hospitals, Cairo University PO: 11451, Kasr Al-Aini street, Cairo Egypt beero4a@yahoo.com

Received on Dez/11/2019 Accepted on Jun/12/2020

DOI: 10.20945/2359-3997000000283 


\section{INTRODUCTION}

$\mathrm{T}$ hyroid nodules are widely encountered in the population. The prevalence of thyroid nodules is increasing (1). They are detected in $2 \%-6 \%$ of the population by palpation, in 19\%-35\% by ultrasonography (US), and in $8 \%-65 \%$ through autopsy data (2). US is the most accurate and cost-effective imaging method in diagnosis of thyroid nodules (2). Ultrasound criteria to differentiate between benign and malignant nodules of the thyroid gland have been the point of research in many scientific papers. Suspicious features of nodules to predict malignancy include hypoechogenicity, microcalcification, taller-than-wide shape, irregular or microlobulated margins, and increased intranodular vascularity (3).

Fine needle aspiration cytology (FNAC) is mandatory in the preoperative diagnosis of thyroid nodules to distinguish benign from malignant nodules (4).

Selection of thyroid nodules for FNAC is still confusing. Apractical thyroid imaging reporting and data system (TIRADS) for thyroid nodules has been proposed to classify nodules of the thyroid gland to solve the problem of nodule selection for FNAC (5).

Real-time elastography is a method used in the evaluation of thyroid nodules by comparing tissue elasticity. Two kinds of elasticity can be assessed by strain elastography. First, colors around and within the nodules are evaluated and visually scored according to the 4-5 scale scoring system. Second, regions of interest are specified as the target region and the adjacent reference region. Later, elastography calculates the strain ratio (SR) automatically. Higher elastography score and SR indicate a high probability of malignancy (6).

\section{MATERIALS AND METHODS}

\section{Patients}

From 2015 to 2018 at Cairo University Hospital, a series of 409 patients with thyroid nodules (373 females and 36 males) with a mean age of $39 \pm 10$ SD was referred for thyroid ultrasound. The study was approved by our institution's Research Ethics Committee, and all patients gave their informed written consent before inclusion in the study.

\section{Thyroid ultrasound}

Ultrasound examination of the neck was done using a Hitachi (Avius) machine (Hitachi Medical Corporation). Neck ultrasound examination was done with the patient lying in a supine position, with the neck slightly extended by placing a pillow under the patient's shoulders. The scanning protocol in our study included scanning of the thyroid gland in both transverse and longitudinal planes by brightness mode (B-mode), color-coded Doppler imaging (CCDI) and power Doppler imaging (PDI), and real-time elastography and SR.

\section{Image analysis}

Ultrasound and power Doppler images were analyzed. We did not know the other clinical and final pathological reports; the ultrasound operator was blinded to clinical data and to the pathological results. The thyroid nodules detected in this study were analyzed according to their number (multinodular goiter [MNG] or solitary thyroid nodule), echogenicity (nodules could be "hyperechoic," "isoechoic," "hypoechoic," "marked hypoechoic," heterogeneous, cystic, or complex cystic), borders (regular or irregular), presence of calcification, and type of calcification (egg shell, microcalcification, or punctate). Other parameters of the nodules were recorded including anteroposterior diameter/transverse ratio ("taller-than-wide," $\geq 1 \mathrm{~mm}$, and "wider-than-tall," $<\mathrm{lmm}$ ), presence of breakdown, or a surrounding halo.

Categorization of each nodule according to the European TIRADS was from 1 to 5 : TIRADS $1=$ normal thyroid gland; TIRADS2 = thyroid gland has a simple cyst, spongiform cyst, or isolated macrocalcification, or is a diffuse hypoechoic enlarged thyroid gland; TIRADS3 $=$ has isoechoic or hyperechoic nodule and has no high suspicious US features; TIRAD4 = has moderately hypoechogenic nodule and has no high suspicious US features; and TIRADS 5 = has at least one of the high suspicious US features and/or adenopathy (Figure 1). Features of high suspicion are irregular shape, irregular margins, microcalcification, and marked hypoechogenicity (and solid) (7).

Strain elastograms of nodules are qualitatively evaluated with a stepwise scoring system, according to the prevalent color in the nodule. We used the scoring system based on the breast strain USE scale of Itoh and cols. It includes four different patterns. Thyroid nodules with scores 1 and 2 are considered benign, and those with scores 3 and 4 (Figure 2) are classified as suspicious for malignancy (7).

The semiquantitative score of elastography was represented by the SR method. Two areas were selected: area $\mathrm{A}$, representing the region of interest, and area $\mathrm{B}$, 

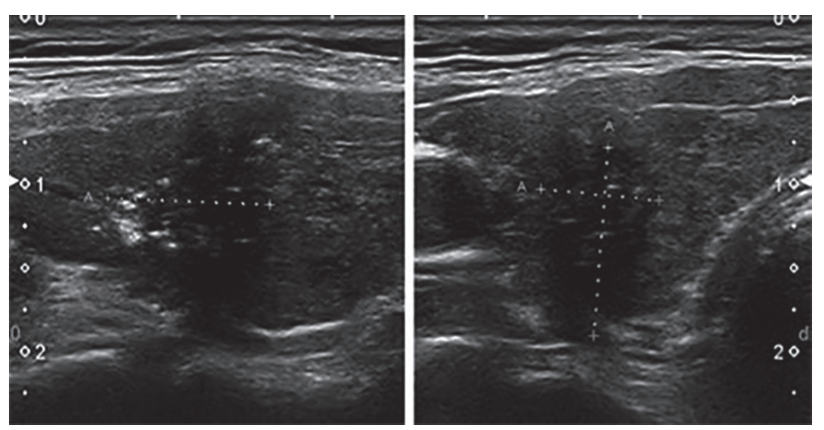

Figure 1. Markedly hypoechoic nodule with irregular outline, AP/T diameter $>1$ and microcalcifications (TIRADS 5).

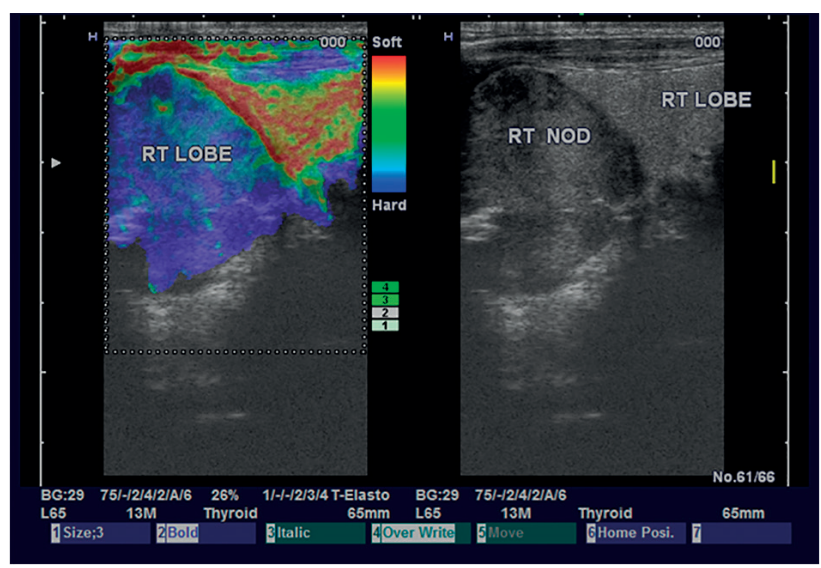

Figure 2. Malignant right lobe nodule with elastography score 4.

representing the normal area. Area $\mathrm{B}$ was then divided by area $\mathrm{A}$. For masses with a homogeneous pattern of elasticity, area A was chosen from any region, but in those with heterogeneous patterns, area A was chosen to cover all heterogeneous areas as much as possible. Both areas were manually selected according to these criteria. Additionally, multiple measures of SR were taken, and the median of these measures was recorded and considered for statistical analysis. Subsequently, the best cutoff value was calculated and was used for the calculation of diagnostic value.

All our data were gathered by a single expert operator, who was blinded to any clinical or cytopathological findings to give the best results and avoid bias.

The final diagnosis was reached by histopathological examination of the thyroid after thyroidectomy in $14 \%$ $(57 / 409)$ and by US-guided FNAC in $86 \%(352 / 409)$.

\section{Statistical analysis}

Data management and analysis were performed using the Statistical Package for Social Sciences (SPSS) version 25. Numerical data were summarized using means and standard deviations or medians and/or ranges, as appropriate. Categorical data were summarized as numbers and percentages. Estimates of the frequency were done using the numbers and percentages. Numerical data were explored for normality using the Kolmogorov-Smirnov test and Shapiro-Wilk test.

To measure associations between categorical variables, a chi-square test or Fisher's exact test was used to compare between two independent percentages.

Odds ratios (ORs) and 95\% confidence intervals (95\% CIs) were also used (a 95\% CI that did not contain 1.0 was considered significant). McNemar's test was used to compare between two dependent percentages. Kappa statistics were used to test for agreement between categorical variables. Comparisons between two groups for normally distributed numeric variables were made using Student's t-test, while for non-normally distributed numeric variables, comparisons were made using the Mann-Whitney $U$ test. Logistic regression was done to give the adjusted OR and magnitude of the effect of different factors. All tests were two tailed, and a $\mathrm{p}$ value $\leq 0.05$ was considered significant. The receiver operating characteristic curve (ROC curve) was used to determine the best cutoff point, sensitivity, specificity, and area under the curve (AUC).

\section{RESULTS}

Our study included 409 patients with thyroid nodules. Their mean age was 39 years \pm 10 SD; 36 were males and 373 were females. Their nodules were classified according to their number into $\mathrm{MNG}$ in 323 cases and solitary thyroid nodule in 86 cases. Regarding echogenicity, 47 were isoechoic, 78 were hyperechoic, 257 were hypoechoic, 8 were marked hypoechoic, 14 were heterogeneous, 2 were cystic, and 3 were complex cystic. Calcifications were found in 100 cases: 6 punctate calcifications and 94 microcalcifications. We found 159 cases with breakdown changes inside the nodule and a halo sign in 260 cases. The anteroposterior/transverse ratio was $<1 \mathrm{~mm}$ in 398 cases and $\geq 1 \mathrm{~mm}$ in 11 cases. Real-time elastography revealed that there were 45 cases with score 1,316 with score 2, 42 with score 3 , and 6 with score 4 . According to the TIRADS classification, there were 3 TIRADS 2 cases, 100 TIRADS 3 cases, 196 TIRADS 4 cases, and 110 TIRADS 5 cases. The final diagnosis was 22 malignant nodules and 387 benign nodules. 
The characteristics of benign $(\mathrm{n}=387)$ and malignant $(\mathrm{n}=22)$ nodules are listed in Table 1 .

No significant differences were determined between the benign and malignant groups in terms of nodule site, number of nodules, and presence of breakdown changes. The anteroposterior/transverse ratio, degree of echogenicity, border, presence of calcification, absence of halo sign, TIRADS classification, and SR were found to be significantly different between benign and malignant nodules as in Table 2 .

The elastic properties of thyroid nodules showed to be a good discriminator between malignant and benign nodules $(\mathrm{P}<.001)$.

ROC curve analysis was performed to determine the cutoff point of the SR to discriminate between benign and malignant thyroid nodules (Figure 3). Sensitivity of $95.2 \%$, specificity of $86.5 \%, 27.8 \%$ positive predictive value (PPV), and $99.7 \%$ negative predictive value (NPV)were achieved. An AUC of 0.96 and a cutoff value of $>2.32$ with $p$ value $<0.001$ were determined as demonstrated in Table 3 .

\section{Multivariate analysis (logistic regression for prediction of malignancy in thyroid nodules in Table 4)}

Patients with irregular borders have nearly 17 times increased risk of malignancy than those with regular borders.

For every unit increase in SR, the risk of malignancy increases by nearly 2 times.

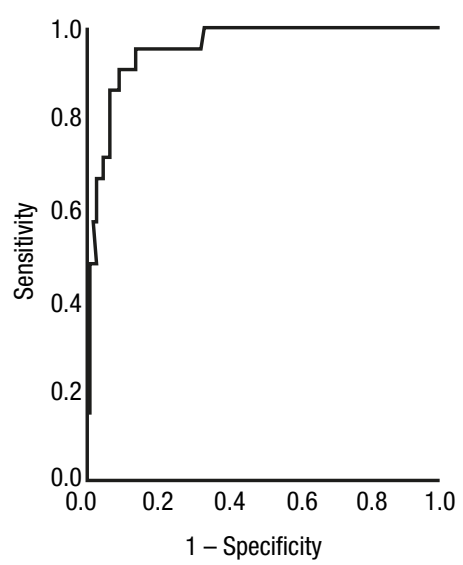

Figure 3. Receiver operating characteristic (ROC) curve analysis to determine cut off point of the strain ratio to discriminate between benign and malignant thyroid nodule.
Table 1. The characteristic of benign and malignant nodules

\begin{tabular}{|c|c|c|c|c|c|}
\hline \multirow{3}{*}{ Factors } & \multicolumn{4}{|c|}{ Biopsy } & \multirow{3}{*}{$P$ value } \\
\hline & \multicolumn{2}{|c|}{ Malignant } & \multicolumn{2}{|c|}{ Benign } & \\
\hline & $\mathrm{n}=\mathbf{2 2}$ & Row \% & $\mathbf{n}=\mathbf{3 8 7}$ & Row \% & \\
\hline \multicolumn{6}{|l|}{ Site } \\
\hline Isthmic thyroid nodule & 0 & 0.0 & 9 & 100.0 & 1 \\
\hline Right thyroid nodule & 12 & 5.7 & 197 & 94.3 & \\
\hline Left thyroid nodule & 10 & 5.2 & 181 & 94.8 & \\
\hline \multicolumn{6}{|l|}{ Elastography } \\
\hline 1 & 0 & 0.0 & 45 & 100.0 & $<0.001$ \\
\hline 2 & 3 & 0.9 & 313 & 99.1 & \\
\hline 3 & 15 & 35.7 & 27 & 64.3 & \\
\hline 4 & 4 & 66.7 & 2 & 33.3 & \\
\hline \multicolumn{6}{|l|}{$\mathrm{AP} / \mathrm{T}$} \\
\hline$<1$ & 13 & 3.3 & 385 & 96.7 & $<0.001$ \\
\hline$\geq 1$ & 9 & 81.8 & 2 & 18.2 & \\
\hline \multicolumn{6}{|l|}{ Number of nodule } \\
\hline MNG & 13 & 4.0 & 310 & 96.0 & 0.019 \\
\hline Solitary thyroid nodule & 9 & 10.5 & 77 & 89.5 & \\
\hline \multicolumn{6}{|l|}{ Nodule character } \\
\hline Dominant nodule & 11 & 3.9 & 271 & 96.1 & 0.053 \\
\hline Small nodule & 2 & 4.9 & 39 & 95.1 & \\
\hline Solitary nodule & 9 & 10.5 & 77 & 89.5 & \\
\hline \multicolumn{6}{|l|}{ Echogenicity } \\
\hline Isoechoic & 0 & 0.0 & 47 & 100.0 & NA \\
\hline Hyperechoic & 0 & 0.0 & 78 & 100.0 & \\
\hline Hypoechoic & 14 & 5.4 & 243 & 94.6 & \\
\hline Marked hypoechoic & 8 & 100.0 & 0 & 0.0 & \\
\hline Heterogenous & 0 & 0.0 & 14 & 100.0 & \\
\hline Cystic & 0 & 0.0 & 2 & 100.0 & \\
\hline Complex cystic & 0 & 0.0 & 3 & 100.0 & \\
\hline \multicolumn{6}{|l|}{ Border } \\
\hline Regular & 9 & 2.3 & 379 & 97.7 & $<0.001$ \\
\hline Irregular & 13 & 61.9 & 8 & 38.1 & \\
\hline \multicolumn{6}{|l|}{ Calcification } \\
\hline No & 10 & 3.2 & 299 & 96.8 & 0.001 \\
\hline Yes & 12 & 12.0 & 88 & 88.0 & \\
\hline \multicolumn{6}{|l|}{ Type of calcification 2} \\
\hline Microcalcifications & 7 & 7.4 & 87 & 92.6 & $<0.001$ \\
\hline Punctate & 5 & 83.3 & 1 & 16.7 & \\
\hline No & 10 & 3.2 & 299 & 96.8 & \\
\hline \multicolumn{6}{|l|}{ Breakdown } \\
\hline No & 17 & 6.8 & 233 & 93.2 & 0.110 \\
\hline Yes & 5 & 3.1 & 154 & 96.9 & \\
\hline \multicolumn{6}{|l|}{ Halo } \\
\hline No & 19 & 12.8 & 130 & 87.2 & $<0.001$ \\
\hline Yes & 3 & 1.2 & 257 & 98.8 & \\
\hline \multicolumn{6}{|l|}{ TIRADS } \\
\hline 2 & 0 & 0.0 & 3 & 100.0 & NA \\
\hline 3 & 0 & 0.0 & 100 & 100.0 & \\
\hline 4 & 4 & 2.0 & 192 & 98.0 & \\
\hline 5 & 18 & 16.4 & 92 & 83.6 & \\
\hline \multicolumn{6}{|l|}{ SR } \\
\hline Median (range) & 5.71 & 7-9.7) & $1.4(0$ & 1-8.7) & $<0.001$ \\
\hline
\end{tabular}

$P$ value $<0.05$ is considered significant. 
Table 2. Shows that anteroposterior diameter, the surrounding halo and the calcification can predict malignancy in thyroid nodules

\begin{tabular}{|c|c|c|c|c|c|c|c|}
\hline \multirow{2}{*}{ Factors } & \multicolumn{2}{|c|}{ Malignant } & \multicolumn{2}{|c|}{ Benign } & \multirow{2}{*}{$P$ value } & \multirow{2}{*}{$\mathrm{OR}$} & \multirow{2}{*}{$95 \% \mathrm{Cl}$ for $\mathrm{OP}$} \\
\hline & $n=22$ & (Row \%) & $n=387$ & (Row \%) & & & \\
\hline \multicolumn{8}{|l|}{ AP/T } \\
\hline$\geq 1$ & 9 & 81.8 & 2 & 18.2 & 0.001 & 133 & $26-679$ \\
\hline$<1$ & 13 & 3.3 & 385 & 96.7 & & & \\
\hline \multicolumn{8}{|c|}{ Calcification } \\
\hline Yes & 12 & 12 & 88 & 88 & 0.001 & 4 & $1.7-9.8$ \\
\hline No & 10 & 3.2 & 299 & 96.8 & & & \\
\hline \multicolumn{8}{|l|}{ Halo } \\
\hline No & 19 & 12.8 & 130 & 87.2 & 0.001 & 12.5 & $3.6-43$ \\
\hline Yes & 3 & 1.2 & 257 & 98.8 & & & \\
\hline
\end{tabular}

$\mathrm{OR}=$ Odds Ratio, $95 \% \mathrm{Cl}$ for $\mathrm{OR}=95 \%$ confidence interval for the $=0$ dds Ratio. P-value $\leq 0.05$ is considered significant.

Table 3. ROC curve to determine cut off point of strain ratio that discriminate between malignant and begin thyroid nodules

\begin{tabular}{lcccccccc}
\hline Variable & Cut off point & Sensitivity (\%) & Specificity (\%) & PPV (\%) & NPV (\%) & AUC & $\begin{array}{c}\text { 95\% Cl } \\
\text { for AUC }\end{array}$ & $\begin{array}{l}\boldsymbol{P} \text { value } \\
\text { SR }\end{array}$ \\
\hline & $>2.32$ & 95.2 & 86.5 & 27.8 & 99.7 & 0.96 & $0.92-0.99$ & $<0.001$ \\
\hline
\end{tabular}

SR: strain ratio; PPV: positive predictive value; NPV: negative predictive value; AUC: area under the curve; Cl: confidence interval. $p$ value $<0.05$ is considered significant.

Table 4. Multivariate analysis (logistic regression for prediction of malignancy in thyroid nodule)

\begin{tabular}{lccccc}
\hline Factors & B & S.E. & P value & OR & 95\% Cl for OR \\
\hline SR & 0.8 & 0.2 & $<0.001$ & 2.3 & $1.6-3.4$ \\
Border & 2.4 & 0.9 & 0.009 & 10.6 & $1.8-62$ \\
Constant & -8.7 & 1.5 & $<0.001$ & $<0.001$ & \\
\hline
\end{tabular}

\section{DISCUSSION}

US is usually the first chosen modality for diagnosing intra-thyroid lesions. There is no single imaging criterion that can reliably distinguish benign from malignant thyroid lesions (8).

Currently, ultrasound elastography can evaluate the tissue elasticity of thyroid nodules and thus enhance the diagnostic ability to detect malignant thyroid diseases. Strain elastography determines tissue elasticity, which is related to its composition and cellularity. This is achieved by operator-dependent external compression of the lesion causing tissue deformation. SR is obtained by dividing the mean strain of the surrounding normal tissue by the mean strain within the lesion. Stiff lesions tend to produce high SRs (9).

The aim of our study was to assess the role of strain elastography and the TIRADS scoring system in discriminating malignant thyroid nodules from benign nodules. It included 409 patients with thyroid nodules, both multinodular lesions and solitary thyroid nodules.
No significant differences were determined between the benign and malignant groups in terms of nodule site, number of nodules, and presence of breakdown changes. On the other hand, the anteroposterior/ transverse ratio, the degree of echogenicity, the border (with irregular borders showing nearly 17 times increased risk of malignancy), the presence of calcification, the absence of halo sign, and the TIRADS classification were found to be significantly different between benign and malignant nodules.

The results of the current study agree with a series done by Cooper and cols. (10), who reported that irregular margins, microcalcifications, marked hypoechogenicity, taller-than-wide shape, absent halo sign, and intranodular vascularity are the ultrasonographic features most predictive of malignancy. Furthermore, Mohamed and Abodewan (11) stated that ill-defined margins, spot microcalcifications, and anteroposterior/transverse diameter more than $1 \mathrm{~cm}$ were the most predictive of malignancy. 
In our study, the SR was found to be significantly different between benign and malignant nodules, and the elastic properties of thyroid nodules showed to be a good discriminator $(\mathrm{P}<0.001)$. SR with a cutoff value of 2.23 was found to have a sensitivity of $95.2 \%$, specificity of $86.5 \%, 27.8 \% \mathrm{PPV}$, and $99.7 \% \mathrm{NPV}$ to discriminate between benign and malignant thyroid nodules. Interestingly, we found that for every unit increase in SR, the risk of malignancy increased by nearly 2 times. However, the low specificity might be due to the small number of included malignant nodules (22 nodules), so further studies including a higher number of malignant nodules are recommended.

Our results are in agreement with those of Sachdev and cols., who evaluated 100 thyroid nodules using ultrasound TIRADS classification and performed strain elastography using elasticity score and SR. Strain elastography had high sensitivity and specificity for differentiating malignant from benign thyroid nodules. The majority of benign thyroid nodules had elasticity scores of 1 and 2, while the majority of malignant thyroid nodules had elasticity scores of 3 and 4 (9). Gay and cols. demonstrated that strain elastography correlated significantly with histological outcomes in indeterminate cytology thyroid nodules (12).

Bhatia and cols. reported that the difference was most statistically significant using an elasticity score > 2 to predict malignancy, which achieved $75 \%$ accuracy (75\% sensitivity, $74 \%$ specificity) (13). Rubaltelli and cols., in their study on 40 patients, observed a sensitivity and specificity of $81.8 \%$ and $87.5 \%$, respectively, which are slightly higher than those of this study (14).

Analysis of a database of more than 3000 thyroid nodules that was recorded in a study sponsored by the Society of Radiologists in Ultrasound showed that no more than $2 \%$ of TIRADS1 and TIRADS 2 nodules, $5 \%$ of TIRADS 3 nodules, $5 \%$ to $20 \%$ of TIRADS 4 nodules, and at least $20 \%$ of TIRADS 5 nodules carry malignant risk (15).

Regarding SR, the results varied. Cantisani and cols. reported that an SR greater than 2 predicted malignancy with a sensitivity of $86 \%$ and a specificity of $82 \%$, whereas in another study, Ding and cols. determined a cutoff point of 2.73 with a sensitivity of $89.3 \%$ and specificity of $73.2 \%(16,17)$.

Dawoud and Dawoud found that gray scale, color Doppler US, and elastography combined were more sensitive and accurate than US features alone in prediction of malignancy of solitary thyroid nodules, with a sensitivity of $94.12 \%$, specificity of $76.74 \%$, and accuracy of $81.67 \%$ in 60 patients with solitary thyroid nodules. They also found that the elastography score of malignant nodules (score 3 and 4) was found in 10 $(58.8 \%)$ of 17 malignant nodules and $9(20.9 \%)$ of 43 benign nodules, with a sensitivity of $58.82 \%$, specificity of $79.07 \%$, and accuracy of $73.33 \%$ (18).

Asteria and cols. (19) reported that elastosonography had a sensitivity of $94.1 \%$, specificity of $81 \%, 55.2 \%$ PPV, and 98.2\%NPVfor thyroid cancer diagnosis, while the accuracy was $83.7 \%$. Rago and cols. (20) showed sensitivity and specificity as high as $97 \%$ and $100 \%$, respectively, using US elastography, while Ferdous and cols. (21) reported a sensitivity of the elastography score of $84 \%$, while the specificity was $84.7 \%$, the PPV was $70 \%$, the NPV was $92.6 \%$, and the accuracy was $84.5 \%$. Suspicious US findings (TIRADS4 and 5) combined with suspicious elastography score (3 and 4) were found in $16(94.1 \%)$ of 17 malignant nodules, with $94.12 \%$ sensitivity, $76.74 \%$ specificity, and $81.67 \%$ accuracy.

Through logistic regression analysis using 12 different sonographic features of thyroid nodules (margin, border, shape, echogenicity, calcification, posterior acoustic halo, acoustic halo at the periphery of the nodule, capsule of thyroid, vascularity, suspected cervical lymph node metastasis, elastography score, and hypoenhancement pattern), Tiantian and cols. concluded that the conventional US, strain elastography, and contrast enhanced US is an effective and accurate diagnostic tool for differentiating malignant and benign thyroid nodules (22).

In conflict with our findings, Schenke and Zimny conducted a study including 244 thyroid nodules and analyzed the visual elasticity scores, strain value (SV), and TIRADS classification and found that the sensitivity, specificity, PPV, and NPV of TIRADS were superior to those of sonoelastography. Their data demonstrate that a high TIRADS class alone is predictive for thyroid carcinoma, and the clinical relevance of sonoelastography is negligible (23). Horvath and cols. reported a sensitivity and specificity for TIRADS classification of $63.64 \%$ and $92.31 \%$, respectively (24). Chandramohan and cols. reported low sensitivity and specificity of sonoelastography score, at $72 \%$ and $68 \%$, respectively $(25)$.

In a study comparing strain elastography to Acoustic Radiation Force Impulse (ARFI) imaging, Georgia and cols. showed that strain elastography had low sensitivity and specificity compared to ARFI 
( 0.86 vs. $0.51, p=0.008)$. The reason could be that the elastography is operator dependent and can be affected by several factors such as the experience of the examiner, characteristics of nodules, and artifacts such as carotid artery pulsations (26).

In our study, 2 out of 22 malignant nodules had TIRDAS 4 and 5 scores, with longitudinal axes of $14 \mathrm{~mm}$ and $10 \mathrm{~mm}$, respectively. They were considered borderline size according to the TIRADS recommendations for FNAC. As they had elasticity scores of 4 and 5 with high SRs (6.78 and 9.68, respectively), they were justified for FNA and not follow-up.

In conclusion, elastography and SR proved to be of high significant value in discriminating benign from malignant nodules, so we recommend adding it to the TIRADS classification. Furthermore, while the TIRADS can be used for selection of high-risk nodules; ultrasound elastography can be employed to differentiate benign nodules from suspicious ones. Subsequently, FNAC can be performed with greater confidence and efficiency.SR will be beneficial in borderline cases, such as in approximately $20 \mathrm{~mm}$ TIRADS 3, $15 \mathrm{~mm}$ TIRADS 4 , or $10 \mathrm{~mm}$ TIRADS 5 nodules. FNA will be more favorable than follow-up if such borderline nodules have a high elasticity score or SR above the cutoff level (2.32).

Recommendations: additional studies with a greater variety of malignant nodules in a large series will be required to establish the strain elastography imaging characteristics for different pathological types of thyroid carcinoma.

Acknowledgements: we would like to acknowledge our great Kasr Al Ainy Hospital and its workers, nurses, and staff members for all the support and help in this study and throughout our careers.

Ethical consideration: the study was approved by the institutional ethics committee and form review board of Kasr Al Ainy Hospital. Oral and written informed consent was obtained from all subjects or from their eligible relatives.

The medical record profession has its own code of ethics that applies to all medical record practitioners. Confidentiality of data, safe data storage, and privacy rights are respected by all who handle patient information. Data were coded and patient names or identity did not appear on any of the data collection forms or during statistical analysis.

The manuscript has been read and approved by all the authors and each author believes that the manuscript represents honest work.
Funding: the authors received no funding for this study. No source(s) of support in the form of grants, equipment, drugs.

Disclosure: no potential conflict of interest relevant to this article was reported.

\section{REFERENCES}

1. Niedziela M. Thyroid nodules. Best Pract Res Clin Endocrinol Metab. 2014;28:245-77.

2. Dean DS, Gharib H. Epidemiology of thyroid nodules. Best Pract Res Clin Endocrinol Metab. 2008;22(6):901-11.

3. Carneiro-Pla D. Ultrasound elastography in the evaluation of thyroid nodules for thyroid cancer. Curr Opin Oncol. 2013;25:1-5.

4. Lin JD, ChaoTC, Huang BY, Chen ST, Chang HY, Hsueh C. Thyroid cancer in the thyroid nodules evaluated by ultrasonography and fine-needle aspiration cytology. Thyroid. 2005;15(7):708-17.

5. Lim DJ, Luo S, Kim MH, Ko SH, Kim Y. Interobserver agreement and intraobserver reproducibility in thyroid ultrasound elastography. Am J Roentgenol. 2012;198(4):896-901.

6. Russ G, Royer B, Bigorgne C, Rouxel A, Bienvenu-Perrard M, Leenhardt L. Prospective evaluation of thyroid imaging reporting and data system on 4550 nodules with and without elastography. Eur J Endocrinol. 2013;168(5):649-55.

7. Itoh A, Ueno E, Tohno E, Kamma H, Takahashi $H$, Shiina T, et al. Breast disease: clinical application of US elastography for diagnosis. Radiology. 2006;239(2):341-50.

8. Soto GD, Halperin I, Squarcia M, Lomeña F, Domingo MP. Update in thyroid imaging. The expanding world of thyroid imaging and its translation to clinical practice. Hormones. 2010;9(4):287-98.

9. Sachdev N, Sana SG, Prasad A. Clinical utility of TIRADS and ultrasound elastography in characterization of thyroid nodules. Int J Res Med Sci. 2019;7(2):328.

10. Cooper DS, Doherty GM, Haugen BR, Kloos RT, Lee SL, Mandel SJ, et al. Revised American Thyroid Association management guidelines for patients with thyroid nodules and differentiated thyroid cancer: the AmericanThyroid Association (ATA) guidelines taskforce on thyroid nodules and differentiated thyroid cancer. Thyroid. 2009;19(11):1167-214.

11. Mohamed RE, Abodewan KA. Diagnostic utility of real-time ultrasound elastography for prediction of malignancy in solid thyroid nodules. Egyptian Journal of Radiology and Nuclear Medicine. 2013;44(1):33-43.

12. Gay S, Schiaffino S, Santamorena G, Massa B, Ansaldo G, Turtulici G, et al. Role of Strain Elastography and Shear-Wave Elastography in a Multiparametric Clinical Approach to Indeterminate Cytology Thyroid Nodules. Med Sci Monit. 2018;24:6273-9.

13. Bhatia KS, Rasalkar DP, Lee YP, Wong KT, King AD, Yuen HY, et al. Cystic change in thyroid nodules: a confounding factor for realtime qualitative thyroid ultrasound elastography. Clin Radiol. 2011;66(9):799-807.

14. Rubaltelli L, Corradin S, Dorigo A, Stabilito M,Tregnaghi A, Borsato $S$, et al. Differential diagnosis of benign and malignant thyroid nodules at elastosonography. Ultraschall Med. 2009;30(02):175-9.

15. Grant EG, Tessler FN, Hoang JK, Langer JE, Beland MD, Berland LL, et al. Thyroid ultrasound reporting lexicon: white paper of the ACR thyroid imaging, reporting and data system (TIRADS) committee. J Am Coll Radiol. 2015;12(12):1272-9.

16. Cantisani V, Lodise P, Grazhdani H, Mancuso E, Maggini E, Di Rocco G, et al. Ultrasound elastography in the evaluation of thyroid pathology. Current status. Eur J Radiol. 2014;83(3):420-8.

17. Ding J, Cheng $\mathrm{H}$, Ning C, Huang J, Zhang Y. Quantitative measurement for thyroid cancer characterization based on elastography. J Ultrasound Med. 2011;30(9):1259-66. 
18. Dawoud MM, Dawoud RM. Added value of strain elastosonography in prediction of malignancy in solitary thyroid nodule. Egyptian Journal of Radiology and Nuclear Medicine. 2017;48(4):905-12.

19. Asteria C, Giovanardi A, Pizzocaro A, Cozzaglio L, Morabito A, Somalvico $\mathrm{F}$, et al. US-elastography in the differential diagnosis of benign and malignant thyroid nodules. Thyroid. 2008;18(5):523-31.

20. Rago T, Scutari M, Santini F, Loiacono V, Piaggi P, Di Coscio G, et al. Real-time elastosonography: useful tool for refining the presurgical diagnosis in thyroid nodules with indeterminate or nondiagnostic cytology. J Clin Endocrinol Metab. 2010;95(12):5274-80.

21. Ferdous J, Ur Rahman M, Rahman HA, Hossain J, Sharmin S, Yasmin F, et al. Efficacy of Sonoelastography Images in Solitary Cold Thyroid Nodule and Compared with Fine Needle Aspiration Cytology Findings (FNAC). Bangladesh Journal of Nuclear Medicine. 2016;19(2):103-6.

22. Pang $T$, Huang $L$, Deng $Y$, Wang $T$, Chen $S$, Gong $X$, et al. Logistic regression analysis of conventional ultrasonography, strain elastosonography, and contrast-enhanced ultrasound characteristics for the differentiation of benign and malignant thyroid nodules. PLoS One. 2017;12:e0188987.

23. Schenke S, Zimny M. Combination of sonoelastography and TIRADS for the diagnostic assessment of thyroid nodules. Ultrasound Med Biol. 2018;44(3):575-83.

24. Horvath E, Silva CF, Majlis S, Rodriguez I, Skoknic V, Castro A, et al. Prospective validation of the ultrasound basedTIRADS (Thyroid Imaging Reporting And Data System) classification: results in surgically resected thyroid nodules. Eur Radiol. 2017;27(6):2619-28.

25. Chandramohan A, Khurana A, Pushpa BT, Manipadam MT, Naik D, Thomas $\mathrm{N}$, et al. IsTIRADS a practical and accurate system for use in daily clinical practice? Indian J Radiol Imaging. 2016;26:145-52.

26. Kyriakidou G, Friedrich-Rust M, Bon D, Sircar I, Schrecker C, Bogdanou D, rt al. Comparison of strain elastography, point shear wave elastography using acoustic radiation force impulse imaging and 2D-shear wave elastography for the differentiation of thyroid nodules. PloS One. 2018;13:e0204095. 


\section{Role of elastography strain ratio and TIRADS score in predicting malignant thyroid nodule}

DOI: 10.20945/2359-3997000000283

Arch Endocrinol Metab. 2020;64(6):735-42

Where you read (AUTHORS):

Hussein Hassan Okasha ${ }^{1}$ https://orcid.org/0000-0002-0815-1394

Mona Mansor ${ }^{1}$

https://orcid.org/0000-0002-7274-5429

Nermine Sheriba ${ }^{2}$

https://orcid. org/0000-0002-1222-8948

Maha Assem ${ }^{1}$

https://orcid org/0000-0003-3295-2586

Yasmine Abdelfattah

https://orcid.org/0000-0002-2465-3757

Omar A. Ashoush ${ }^{3}$

https://orcid.org/0000-0003-3992-9856

Maha Rakha ${ }^{3}$

https://orcid. org/0000-0002-8287-7599

Dalia Abdelfattah 4

https://orcid.org/0000-0002-8598-7128

Shereen Sadik El- Sawy ${ }^{2}$ https://orcid.org/0000-0001-9642-4657

Mai Elshenoufy ${ }^{1}$

https://orcid. org/0000-0002-9781-9463

Ahmed Amr Mohsen ${ }^{5}$

https://orcid.org/0000-0003-3893-1524

Heba Kamal Sedrak ${ }^{1}$

https://orcid. org/0000-0001-9143-3247

Abeer Awad Abdellatif ${ }^{1}$ https://orcid. org/0000-0001-9945-9767

Should read:

Hussein Hassan Okasha

https://orcid. org/0000-0002-0815-1394

Mona Mansor ${ }^{1}$

https://orcid. org/0000-0002-7274-5429

Nermine Sheriba ${ }^{2}$

https://orcid. org/0000-0002-1222-8948

Maha Assem ${ }^{1}$

https://orcid. org/0000-0003-3295-2586

yasmine abd elfatah

https://orcid org/0000-0002-5302-4087

Omar A. Ashoush'

https://orcid. org/0000-0003-3992-9856

Maha Rakha ${ }^{1}$

https://orcid org/0000-0002-8287-7599

Dalia Abdelfatah ${ }^{3}$

https://orcid.org/0000-0002-8598-7128

Shereen Sadik El-Sawy

https//orcid org/0000-0001-9642-4657

Mai Elshenoufy ${ }^{1}$

https://orcid org/0000-0002-9781-9463

Ahmed Amr Mohsen 4

https://orcid.org/0000-0003-3893-1524

Heba Kamal Sedrak ${ }^{1}$

https://orcid.org/0000-0001-9143-3247

Abeer Awad Abdellatif ${ }^{1}$

https://orcid.org/0000-0001-9945-9767

DOI: 10. 20945/2359-3997000000328

120
1 Kasr Al-Aini Hospitals, Cairo University, Cairo, Egypt

${ }^{2}$ Ain Shams University, Cairo, Egypt

${ }^{3}$ Kasr Alainy Faculty of Medicine,

Cairo University, Cairo, Egypt

${ }^{4}$ Biostatistic and Cancer

Epidemiology Department,

National Cancer Institute, Cairo

University, Cairo, Egypt

${ }^{5}$ National Research

Centre, Cairo, Egypt
${ }^{1}$ Kasr Al-Aini Hospitals, Cairo

University, Cairo, Egypt

${ }^{2}$ Ain Shams University, Cairo, Egypt

${ }^{3}$ Biostatistic and Cancer

Epidemiology Department,

National Cancer Institute, Cairo

University, Cairo, Egypt

${ }^{4}$ National Research

Centre, Cairo, Egypt 